\title{
A Broadband Transmission Metasurface with Polarization-Transforming Functionality
}

\author{
Wei CHEN, Jun GAO, Xiang-Yu CAO, Huan-Huan YANG, \\ Zhao ZHANG, Si-Jia LI, Jiang-Feng HAN, Chen ZHANG \\ Information and Navigation College, Air Force Engineering University, No.1 Feng Hao Road, Xi'an 710077, China \\ 1722020353@qq.com,gjgj9694@163.com,xiangyucaokdy@163.com, jianye8901@126.com, \\ bjzhangzhao323@126.com,1sj051@126.com, ghwewehan@126.com, xue320long@sina.cn
}

Submitted April 4, 2017 / Accepted July 15, 2017

\begin{abstract}
A transmission metasurface with polarizationtransforming functionalities is presented. The unit cell is characterized by broad band, low profile and low loss. Due to the asymmetric structure, the proposed metasurface shows different responses to different linear-polarized incidences. For a particular linear-polarized incidence, the polarization direction of transmitted waves will be rotated by $90^{\circ}$ in $3.72-8.28 \mathrm{GHz}$ with high polarization conversion ratio (PCR). However, for the orthogonal-polarized ones, the incidence will be reflected totally and the polarization remains. The operating mechanism of the proposed metasurface is analyzed both by theoretical investigations and numerical simulations. Measured results of the fabricated samples are in good agreement with the simulated ones.
\end{abstract}

\section{Keywords}

Metasurface, broadband, polarization-dependent, high polarization conversion ratio

\section{Introduction}

In recent years, the constantly updating communication technology has wonderfully advanced the living standards of human beings, and the upcoming $5 \mathrm{G}$ mobile communication era will be fascinating because of its high information transmission rate. Compared to $4 \mathrm{G}$ communication network, 5G communication system not only is far ahead of the rate, but also significantly improves user density and user capacity based on the polarization isolation characteristics of electromagnetic waves. Polarization, as an important feature of electromagnetic waves, not only has important applications prospects in the field of modern communications, but also in radar, detection, and imaging [1]. Meanwhile, the polarization control is completely required in microwave regime, as well as in terahertz, infrared, and visible frequencies [2]. Therefore, the research of how to manipulate the polarization of electromagnetic wave is in great demands.
The manipulation of the electromagnetic wave by the metamaterials normally aims at the control of its amplitude [3-6], phase [7-11] and polarization [12-22]. According to the propagation direction of electromagnetic waves, polarization-manipulating metamaterials consist of reflective and transmitting types. Currently, lots of polarization rotating reflective metamaterial are reported. By using multi-layer cascading [12], metallized via [13], multiple plasmon resonances [14] and other anisotropic structures, many reflective polarization convertors with broadband and high polarization conversion ratio performance have been successfully presented. The conventional applications of the reflective polarization-manipulating metamaterials include: acting as reflective surface to achieve polarization transformation [15] or implement a reflectarray antenna [16], constituting a reflective screen to reduce radar cross section (RCS) [17]. For transmitting ones, it is usually employed as filters for waveguide transmission systems [18], as polarization convertors for antennas [19] and as antennas' transmitarrays to achieving beam focusing, beam steering and beam shaping [20]. However, the precondition for the application is that these polarization-manipulating metamaterials exhibit low loss, high polarization conversion efficiency and low fabrication cost. But, how to take all these factors into consideration still remains a big challenge for the researches of polarization convertors.

In this paper, a kind of polarization-dependent transmission metasurface is proposed. In the frequency range of $3.72-8.28 \mathrm{GHz}$, the polarization direction of the transmitted wave will be rotated by $90^{\circ}$ when a particular linearpolarized wave irradiates the metasurface. The proposed polarization-transforming metasurface is characterized by broadband, high efficiency, low loss and low profile. Because of these noticeable performances, the proposed metasurface has potential applications in waveguide transmission systems and transmitarray antenna domain.

\section{Structure Design}

The unit cell structure of the polarization-transforming transmission metasurface is shown in Fig. 1(a). The tri- 
(a)

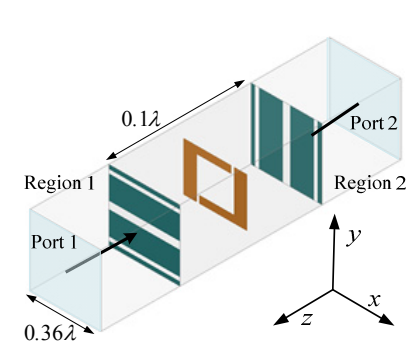

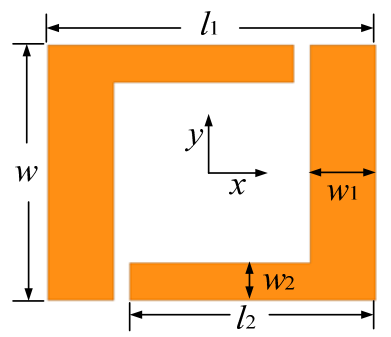

(b)

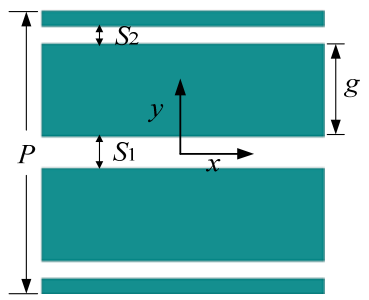

(c)

Fig. 1. Illustration of the presented unit cell:(a) Schematic of the simulation; (b) the RDSRR; (c) the stripe MG with surface slots parallel to $x$-axis.

layers unit cell consists of a rectangle diagonal-split-ring resonator (RDSRR) structure and two orthogonal stripe metal grids (MG). The RDSRR, as shown in Fig. 1(b), is clamped by two substrates, and it is employed by achieving polarization conversion. Two orthogonal MGs, as shown in Fig. 1(c), are etched on the surface of the upper and lower substrate, and are designed to constitute a Fabry-Pérot-like cavity for more efficient polarization conversion [21]. The optimized parameters are as follows: $l 1=16 \mathrm{~mm}, l 2=12$ $\mathrm{mm}, w=12.4 \mathrm{~mm}, w 1=3.2 \mathrm{~mm}, w 2=1.8 \mathrm{~mm}, S 1=2 \mathrm{~mm}$, $S 2=1.1 \mathrm{~mm}, g=5.9 \mathrm{~mm}, P=18 \mathrm{~mm}$. The relative permittivity of the adopted dielectric is 2.65 . The total thickness of the unit cell is $5 \mathrm{~mm}$, approximately $0.1 \lambda$ at $6 \mathrm{GHz}$. During the designing process of the proposed unit cell, Ansoft HFSS 14.0 is used for full-wave simulation. Floquet port and periodic boundary are adopted for a unit cell to simulate an infinite periodic array.

It is worth noting that the proposed metasurface is characterized by polarization-dependent functionalities. When a $y$-polarized electromagnetic wave irradiates from the region 1 , the $y$-polarized incidence can be transformed into the $x$-polarized transmitted wave. And if the polarization direction of the incident wave is parallel to the $x$-axis, the $x$-polarized incidence will be reflected totally, and polarization direction of the reflected wave is also parallel to $x$-axis. However, when the electromagnetic waves irradiate from region 2, the electromagnetic response will be the opposite case.

\section{Simulation and Analysis}

For the sake of analysis, we define $r_{m n}{ }^{\mathrm{xy}}$ as reflection coefficient, and $t_{m n}{ }^{\mathrm{xy}}$ as transmission coefficient. Here $x$ and $y$ represent the electric field directions of the electromagnetic wave, and $m$ and $n$ denote the different regions. For example, $t_{21}{ }^{\mathrm{xy}}$ represents the $x$-polarized field transmit- ted into region 2 when the $y$-polarized plane wave is incident from region 1.

The simulation results are shown in Fig. 2. When the $y$-polarized field is incident from region 1, the results are shown in Fig. 2(a). The curves manifest that, in the frequency range of $3.72-8.28 \mathrm{GHz}, t_{21}{ }^{\mathrm{xy}}$ is greater than $-0.5 \mathrm{~dB}$ and $t_{21}{ }^{\mathrm{yy}}$ is below $-35 \mathrm{~dB}$, which indicates that the polarization direction of transmitted wave is parallel to the $x$-axis; Meanwhile, it is also observed that $r_{11}{ }^{\text {yy }}$ is less than $-10 \mathrm{~dB}$, and $r_{11}{ }^{\mathrm{yy}}$ is less than $-35 \mathrm{~dB}$, which demonstrates that the reflected wave is mainly $y$-polarized. Figure 2(b) shows the simulation results of the $\mathrm{S}$ parameters when the incidence is $x$-polarized. It can be seen that $r_{11}{ }^{\mathrm{xx}}$ is approximately $0 \mathrm{~dB}$. At the same time, both $r_{11}{ }^{\mathrm{yx}}$ and $t_{21}{ }^{\mathrm{xx}}$ are lower than $-35 \mathrm{~dB}$ and $t_{21}{ }^{\mathrm{yx}}$ is less than $-70 \mathrm{~dB}$. These numerical simulations manifest that the $x$-polarized incident waves will be totally reflected when incident from region 1 .

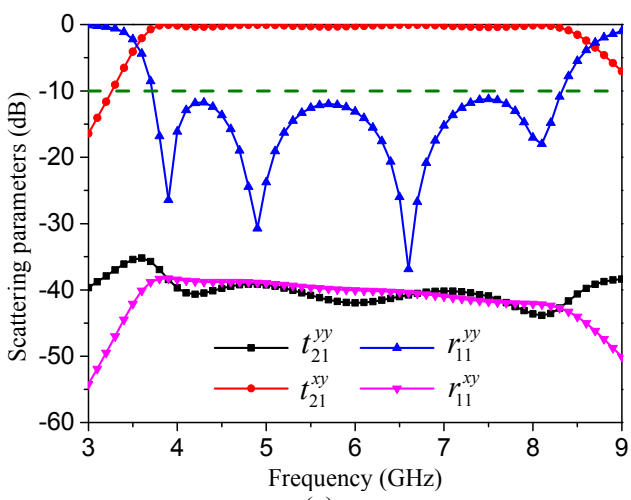

(a)

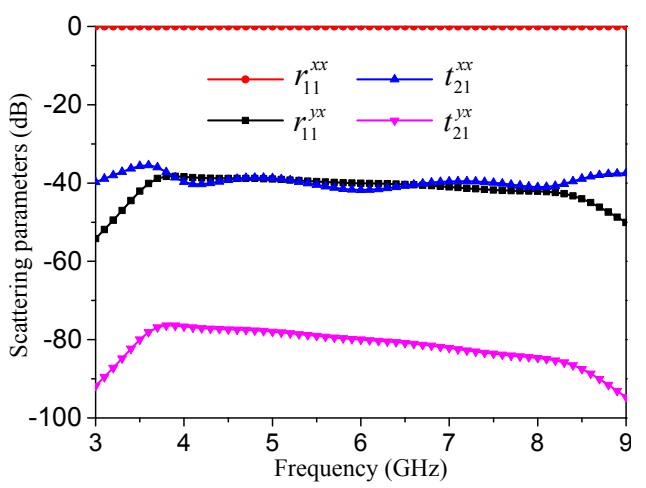

(b)

Fig. 2. Simulation results of the $\mathrm{S}$ parameters when different polarized wave is incident from range 1: (a) $y$-polarized incidence; (b) $x$-polarized incidence.

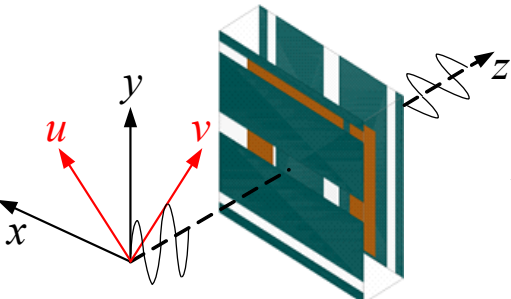

(a)

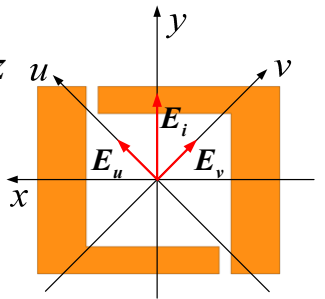

(b)
Fig. 3. Schematic of the incident wave: (a) two different coordinate system; (b) decomposition of $y$-polarized incident wave along $u$-axis and $v$-axis. 
To explain the operating mechanism of the metasurface, we rotate the $x y$-coordinate system by $45^{\circ}$ and thus the uv-coordinate system is obtained, as shown in Fig. 3.

When the $y$-polarized incidence propagates along the z-axis, the electric field can be expressed as $\boldsymbol{E}_{\mathrm{i}}=E_{\mathrm{i}} \exp (\mathrm{j} k z) \boldsymbol{y}$. The electric field of the incident wave can be decomposed into two components along $u$ axis and $v$ axis as:

$$
\boldsymbol{E}_{\mathrm{i}}=\frac{\sqrt{2}}{2} E_{\mathrm{i}} \exp (\mathrm{j} k z) \boldsymbol{u}+\frac{\sqrt{2}}{2} E_{\mathrm{i}} \exp (\mathrm{j} k z) \boldsymbol{v} .
$$

Then the electric field of the reflective and transmitted wave can be presented as (2) and (3), respectively.

$$
\begin{aligned}
\boldsymbol{E}_{\mathrm{r}} & =\frac{\sqrt{2}}{2}\left\{r_{\mathrm{uu}} E_{\mathrm{i}} \exp \left[\mathrm{j}\left(k z+\varphi_{\mathrm{uu}}\right)\right]+r_{\mathrm{uv}} E_{\mathrm{i}} \exp \left[\mathrm{j}\left(k z+\varphi_{\mathrm{uv}}\right)\right]\right\} \boldsymbol{u} \\
& +\frac{\sqrt{2}}{2}\left\{r_{\mathrm{vu}} E_{\mathrm{i}} \exp \left[\mathrm{j}\left(k z+\varphi_{\mathrm{vu}}\right)\right]+r_{\mathrm{vv}} E_{\mathrm{i}} \exp \left[\mathrm{j}\left(k z+\varphi_{\mathrm{vv}}\right)\right]\right\} \boldsymbol{v},
\end{aligned}
$$

$$
\begin{aligned}
\boldsymbol{E}_{\mathrm{t}} & =\frac{\sqrt{2}}{2}\left\{t_{\mathrm{uu}} E_{\mathrm{i}} \exp \left[\mathrm{j}\left(k z+\varphi_{\mathrm{uu}}\right)\right]+t_{\mathrm{uv}} E_{\mathrm{i}} \exp \left[\mathrm{j}\left(k z+\varphi_{\mathrm{uv}}\right)\right]\right\} \boldsymbol{u} \\
& +\frac{\sqrt{2}}{2}\left\{t_{\mathrm{vu}} E_{\mathrm{i}} \exp \left[\mathrm{j}\left(k z+\varphi_{\mathrm{vu}}\right)\right]+t_{\mathrm{vv}} E_{\mathrm{i}} \exp \left[\mathrm{j}\left(k z+\varphi_{\mathrm{vv}}\right)\right]\right\} \boldsymbol{v} .
\end{aligned}
$$

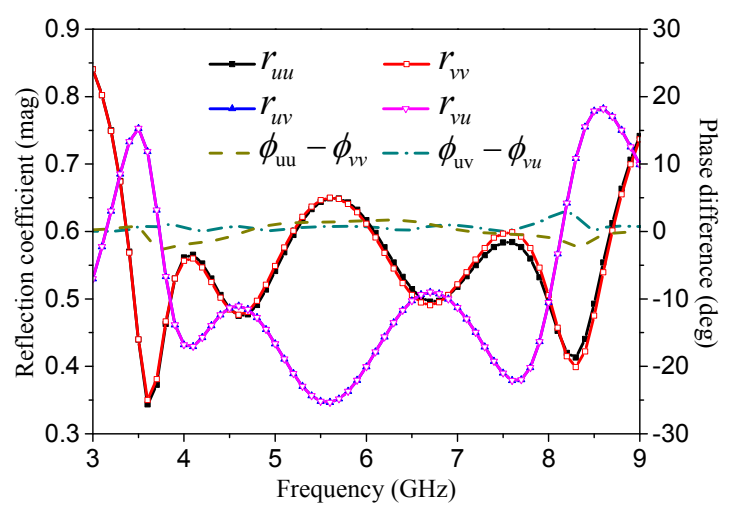

(a)

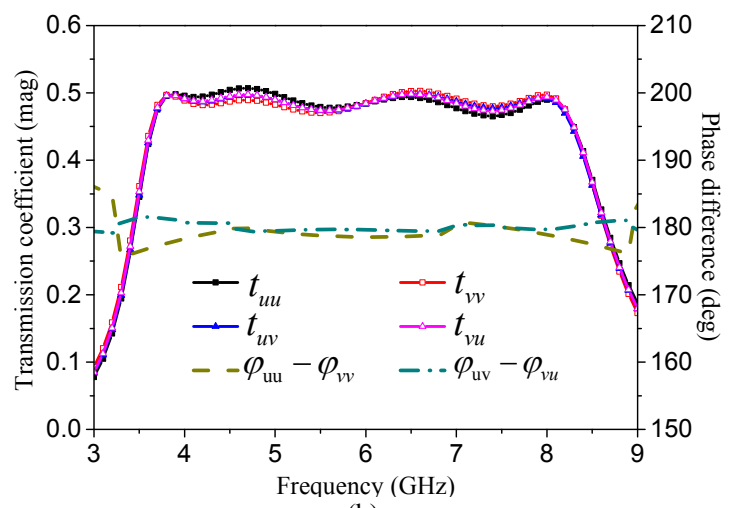

(b)

Fig. 4. Simulation results of the $S$ parameters when the incident wave is parallel to $u$-axis and $v$-axis: (a) reflection coefficients and phase difference; (b) transmission coefficients and phase difference.
Figure 4 shows the $\mathrm{S}$ parameters of the element for $u$ polarized and $v$-polarized incidence. As observed from Fig. 4(a), we can conclude that:

$$
\left\{\begin{array}{c}
r_{\mathrm{uu}}=r_{\mathrm{vv}}=r_{1}, \\
r_{\mathrm{uv}}=r_{\mathrm{vu}}=r_{2}, \\
\Delta \varphi=\varphi_{\mathrm{uu}}-\varphi_{\mathrm{vv}}=0, \\
\Delta \varphi=\varphi_{\mathrm{uv}}-\varphi_{\mathrm{vu}}=0 .
\end{array}\right.
$$

As seen from Fig. 4(b), we can derive that:

$$
\left\{\begin{array}{c}
t_{\mathrm{uu}}=t_{\mathrm{vv}}=t_{1}, \\
t_{\mathrm{uv}}=t_{\mathrm{vu}}=t_{2}, \\
\Delta \varphi=\varphi_{\mathrm{uu}}-\varphi_{\mathrm{vv}}=\pi, \\
\Delta \varphi=\varphi_{\mathrm{uv}}-\varphi_{\mathrm{vu}}=\pi .
\end{array}\right.
$$

By inserting (4) and (5) to (2) and (3), we can get the reflected field $\boldsymbol{E}_{\mathrm{r}}=r_{11}{ }^{\mathrm{yy}} E_{\mathrm{i}} \exp (-\mathrm{j} k z) \boldsymbol{y}$ and the transmitted field $\boldsymbol{E}_{\mathrm{t}}=t_{21}{ }^{\mathrm{xy}} E_{\mathrm{i}} \exp (\mathrm{j} k z) \boldsymbol{x}$. In this case, the reflected field remains $y$-polarized and the transmitted field is twisted to be $x$-polarized. This conclusion coincides well with the simulation results of $y$-polarized incidence, as shown in Fig. 2(a).

Similarly, when the polarization direction of incident wave is parallel to $x$-axis, the reflected field will be $\boldsymbol{E}_{\mathrm{r}}=r_{11}{ }^{\mathrm{xx}} E_{\mathrm{i}} \exp (-\mathrm{j} k z) \boldsymbol{x}$, and the transmitted field is $\boldsymbol{E}_{\mathrm{t}}=r_{21}{ }^{\mathrm{yx}} E_{\mathrm{i}} \exp (\mathrm{j} k z) \boldsymbol{y}$. Therefore, the $x$-polarized reflected wave and the $y$-polarized transmitted field are obtained theoretically. Remarkably, in this occasion, the $x$-polarized incident waves will be reflected totally, just as demonstrated in Fig. 2(b).

Figure 5 exhibits the current distribution of the RDSRR at four peak transmission coefficient frequencies. As the charts show, the RDSRR resembles a pair of symmetrical vibrators. At $3.86 \mathrm{GHz}$, the RDSRR operates like a pair of half-wave oscillator. At $8.04 \mathrm{GHz}$, the RDSRR works corresponding to a pair of full-wave oscillator. Besides, at $4.85 \mathrm{GHz}$ and $6.60 \mathrm{GHz}$, its operating states are between the half-wave and full-wave oscillators. According to the working principle of the symmetrical oscillator, its operation wavelength depends on the arm length of the oscillator. Similarly, the operation wavelength of the proposed metasurface is closely related to the size of the RDSRR. So, based on the same principle, we can obtain the relationship between the unit's operation wavelength $\lambda$ and the arm length $L$ ( $L$ is the sum of long side and short side of the RDSRR). The analytical expression can be depicted as $0.5 \lambda \leq L \leq \lambda$. By calculating, $L \approx l 1+w-w 1-(w 1+w 2) / 2 \approx 22.7 \mathrm{~mm}$. Assume the relative permittivity of the dielectric is denoted by $\varepsilon_{\mathrm{r}}$, the operating frequency range can be calculated approximately:

$$
\frac{c_{0}}{2 L \sqrt{\varepsilon_{\mathrm{r}}}} \leq f \leq \frac{c_{0}}{L \sqrt{\varepsilon_{\mathrm{r}}}} .
$$

The relative permittivity of the dielectric is 2.65 . So, a (4-8) $\mathrm{GHz}$ operating frequency range can be obtained according to (6). This result coincides well with the simulation results. 


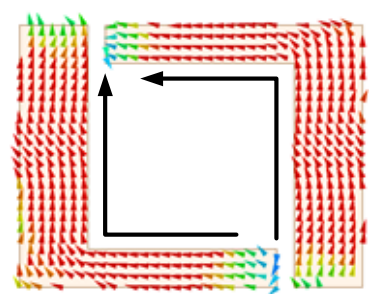

(a)

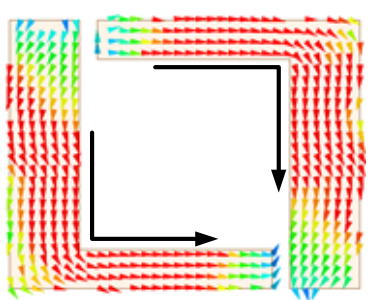

(c)

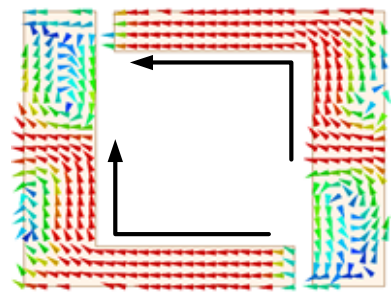

(b)

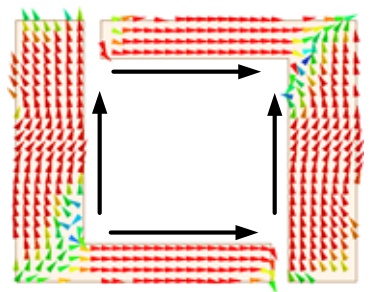

(d)
Fig. 5. The current distribution of the RDSRR at four peak transmission coefficient frequencies: (a) $3.86 \mathrm{GHz}$; (b) $4.85 \mathrm{GHz}$; (c) $6.60 \mathrm{GHz}$; (d) $8.04 \mathrm{GHz}$.

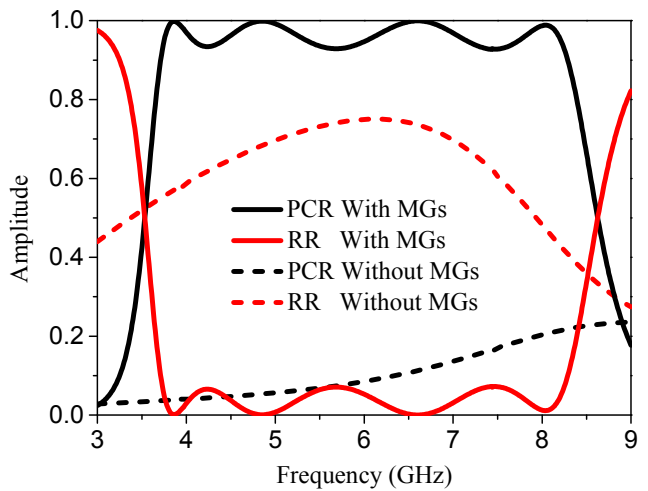

Fig. 6. The PCR and RR simulation results of the metasurface with and without MGs.

To verify functions of the MGs, we also compare the polarization conversion ratio (PCR) and the reflection ratio (RR) of the metasurface with and without the orthogonal MGs. As shown in Fig. 6, the PCR of the metasurface with MGs is greater than 0.9 in the frequency range of 3.72-8.28 GHz. But the PCR of the metasurface without MGs is lower than 0.22 . Besides, in the same frequency range, the RR of the metasurface with MGs is no more than 0.1 , while the RR of the metasurface without MGs will be higher than 0.4. Consequently, it is concluded that the MGs can raise PCR and reduce RR significantly.

\section{Fabrication and Measurement}

A 400 -cell $(20 \times 20)$ metasurface prototype is fabricated and measured. The prototype and measurement setup are illustrated in Fig. 7. During the measurement, two broadband horn antennas are utilized as the emitter and the receiver, respectively. When measuring the reflection coefficient, two horn antennas are on the same side of the sample. When measuring the transmission coefficient, two horn antennas are located on either sides of the prototype.

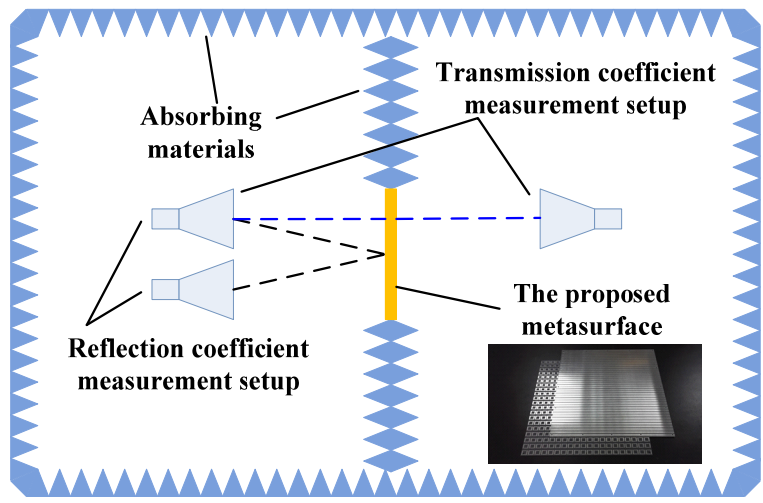

Fig. 7. The prototype and the measurement setup.

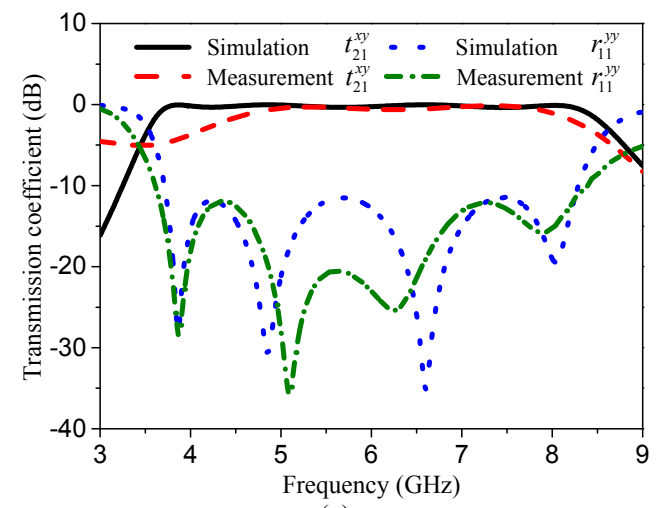

(a)

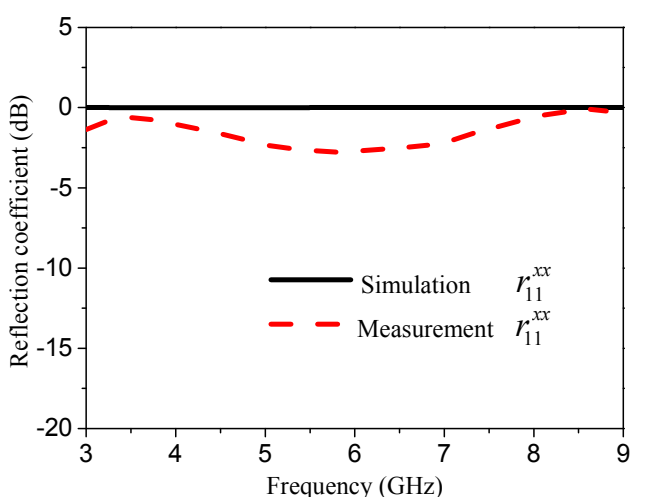

(b)

Fig. 8. The $\mathrm{S}$ parameters comparison between simulation and measurement results when the presented metasurface is irradiated by different polarized incident wave: (a) $y$ polarized incident wave; (b) $x$-polarized incident wave.

Due to the limited measurement conditions, we have only measured $t_{21}{ }^{\mathrm{xy}}$ and $r_{11}{ }^{\mathrm{yy}}$ of the $y$-polarized incidence and $r_{11}{ }^{\mathrm{xx}}$ of the $x$-polarized incidence. The comparison between the measurement results and the simulation ones are shown in Fig. 8.

When the $y$-polarized electromagnetic wave is incident from the region 1 , the transmission coefficient measurement results are shown in Fig. 8(a). It is observed that the measured curves of the cross-polarization transmission coefficient $t_{21}{ }^{\mathrm{xy}}$ coincide well with the simulation ones in the observed frequency range. However, the measurement result has a slight error around $4 \mathrm{GHz}$. The key reason for this consequence is that the size of the sample is finite, 
which is not consistent with the infinite boundary. In addition, the co-polarization reflection coefficient measurement curve is also consistent with the simulation ones. But the measurement curve is lower than the simulation results around $6 \mathrm{GHz}$. By analyzing, the machining error is the main points for this consequence. This inevitable factor also results in the deviation between the measurement and the simulation results of the co-polarization reflection coefficient around $6 \mathrm{GHz}$ when the incidence is $x$-polarized, as shown in Fig. 8(b). In summary, the measurement results confirm the effectiveness of the design.

\section{Conclusion}

In this paper, a polarization-dependent transmission metasurface covering the $\mathrm{C}$ band is presented. The proposed metasurface is characterized by low profile, broadband and high polarization conversion ratio. Numerical simulations demonstrate that, when a particular linear-polarized incidence irradiates the proposed metasurface, the polarization direction of the transmitted wave will be rotated by $90^{\circ}$ in the frequency range of $3.72-8.28 \mathrm{GHz}$. Based on the superposition principle of space wave vector, we have explained the reason of polarization-converting function. At the same time, by analyzing the current distribution of the RDSRR, we have investigated the operation frequency range of the metasurface in detail. Finally, a metasurface prototype is fabricated and measured, and the measurement results are in good agreement with the simulation ones.

\section{Acknowledgments}

Authors thank the supports from the National Natural Science Foundation of China under Grant (No.61471389, No.61501494, and No.61671464). They also thank the reviewers for their valuable comments.

\section{References}

[1] SAFARIPOUR, A., BOWERS, S. M., DASGUPTA, K., et al. Dynamic polarization control of two-dimensional integrated phased arrays. IEEE Transactions on Microwave Theory and Techniques, 2016, vol. 64, no 4, p. 1066-1077. DOI: 10.1109/TMTT.2016.2530704.

[2] WEI, Z., CAO, Y., FAN, Y., et al. Broadband polarization transformation via enhanced asymmetric transmission through arrays of twisted complementary split-ring resonators. Applied Physics Letters, 2011, vol. 99, no. 22, p. 221903-221907. DOI: $10.1063 / 1.3664774$

[3] LI, S. J., GAO, J., CAO, X. Y., et al. Multiband and broadband polarization-insensitive perfect absorber devices based on a tunable and thin double split-ring metamaterial. Optics Express, 2015, vol. 23, no. 3, p. 3523-3533. DOI: 10.1364/OE.23.003523
[4] LI, S. J., GAO, J., CAO, X. Y., et al. Wideband, thin, and polarization-insensitive perfect absorber based the double octagonal rings metamaterials and lumped resistances. Journal of Applied Physics, 2014, vol. 116, p. 043710, DOI: $10.1063 / 1.4891716$

[5] NIROO-JAZI, M., CHAHARMIR, M. R., SHAKER, J., et al. Broadband transmitarray antenna design using polarizationinsensitive frequency selective surfaces. IEEE Transactions on Antennas and Propagation, 2016, vol. 64, no. 1, p. 99-108. DOI: 10.1109/TAP.2015.2500230

[6] ERFANI, E., NIROO-JAZI, M., TATU, S. A high gain broadband gradient index metasurface lens antenna. IEEE Transactions on Antennas and Propagation, 2016, vol. 64, no. 1, p. 1968-1973. DOI: 10.1109/TAP.2016.2526052

[7] ZHAO, Y., CAO, X. Y., GAO, J., et al. Broadband diffusion metasurface based on a single anisotropic element and optimized by the simulated annealing algorithm. Scientific Reports, 2016, vol. 6 , p. 23896. DOI: $10.1038 /$ srep23896

[8] ZHENG, Y. J., GAO, J., CAO, X. Y., et al. Wideband RCS reduction of a microstrip antenna using artificial magnetic conductor structures. IEEE Antennas and Wireless Propagation Letters, 2015, vol. 14, p. 1582-1585. DOI: 10.1109/LAWP.2015.2413456.

[9] LI, H. P., WANG, G. M., XU, H. X., et al. X-band phase-gradient metasurface for high-gain lens antenna application. IEEE Transactions on Antennas and Propagation, 2015, vol. 63, no. 11, p. 5144-5149. DOI: $10.1109 /$ TAP.2015.2475628

[10] CAI, T., WANG, G. M., ZHANG, X. F., et al. Ultra-thin polarization beam splitter using 2-D transmissive phase gradient metasurface. IEEE Transactions on Antennas and Propagation, 2015, vol. 63, no. 12, p. 55629-5636. DOI: 0.1109/TAP.2015.2496115

[11] ZHAO, J. M., SI-MA, B. Y., JIA, N., et al. Achieving flexible lowscattering metasurface based on randomly distribution of metaelements. Optics Express, 2016, vol. 24, no. 24, p. 27849-27857. DOI: $10.1364 /$ OE.24.027849

[12] LI, S. J., CAO, X. Y., XU, L. M., et al. Ultra-broadband reflective metamaterial with RCS reduction based on polarization convertor, information entropy theory and genetic optimization algorithm. Scientific Reports, 2016, vol. 6, p. 37409. DOI: 10.1038/srep37409

[13] JIA, Y. T., LIU, Y., JAY-GUO, Y., et al. Broadband polarization rotation reflective surfaces and their applications to RCS reduction. IEEE Transactions on Antennas and Propagation, 2016, vol. 64, no. 1, p. 179-188. DOI: 10.1109/TAP.2015.2502981

[14] ZHANG, L. B., ZHOU, P. H., LU, H. P., et al. Ultra-thin reflective metamaterial polarization rotator based on multiple plasmon resonances. IEEE Antennas and Wireless Propagation Letters, 2015, vol. 14, p. 1157-1160. DOI: 10.1109/LAWP.2015.2393376

[15] REN, L. S., JIAO, Y. C., LI, F., et al. A dual-layer T-shaped element for broadband circularly polarized reflectarray with linearly polarized feed. IEEE Antennas and Wireless Propagation Letters, 2011, vol. 10, p. 407-410. DOI: 10.1109/LAWP.2011.2148090

[16] MONTORI, S., CACCIAMANI, F., TOMASSONI, C., et al. Novel 1-bit elementary cell for reconfigurable reflectarray antennas. In 41st European Microwave Conference. Manchester (UK), 2011, p. 1288-1291. DOI: 10.23919/EuMC.2011.6101948

[17] CHEN, W., GAO, J., ZHANG, G., et al. A wideband coding reflective metasurface with multiple functionalities. Acta Physica Sinica, 2017, vol. 66, no. 6, p. 064203. DOI: 10.7498/aps.66.064203 (in Chinese)

[18] Barbuto, M., Trotta, F., Bilotti, F., TOSCANO, A. A combined bandpass filter and polarization transformer for horn antennas. IEEE Antennas and Wireless Propagation Letters. 2013, vol. 12, p. 1065-1068. DOI: 10.1109/LAWP.2013.2280151 
[19] ZARIFI, D., ORAIZI, H., SOLEIMANI, M. Improved performance of circularly polarized antenna using semi-planer chiral metamaterial covers. Progress in Electromagnetics Research, 2012, vol. 123, p. 337-354. DOI: 10.2528/PIER11110506

[20] PAN, W. B., HUANG, C., MA, X. L., et al. A dual linearlypolarized transmitarray element with 1-bit phase resolution in Xband. IEEE Antennas and Wireless Propagation Letters, 2014, vol. 14, p. 167-170. DOI: 10.1109/LAWP.2014.2358267

[21] GRADY, N. K., HEYES, J. E., CHOWDHURY, D. R., et al. Terahertz metamaterials for linear polarization conversion and anomalous refraction. Science, 2013, vol. 340, p.1304-1307. DOI: $10.1126 /$ science. 1235399

[22] PFEIFFER, C., GRBIC, A. Bianisotropic metasurfaces for optimal polarization control: analysis and synthesis. Physical Review Applied, 2014, vol. 2, article no. 044011. DOI: 10.1103/PhysRevApplied.2.044011

\section{About the Authors ...}

Wei CHEN was born in China, in 1993, and received the B.S. degree from the School of Information and Navigation, Air Force Engineering University, China, in 2015. Currently, he is working toward M.S. degree at the School of Information and Navigation, Air Force Engineering University. His research interest is in electromagnetic metamaterials and their antenna applications.

Jun GAO received the B.Sc and M.A.Sc degrees from the Air Force Missile Institute, Xi'an, China in 1984 and 1987, respectively. He joined the Air Force Missile Institute in
1987 as an assistant teacher. He became an associate professor in 2000. He is currently a professor of the School of Information and Navigation, Air Force Engineering University of CPLA. He has authored and coauthored more than 60 technical journal articles and conference papers, and holds one China soft patent. His research interests include smart antennas, electromagnetic metamaterials and their antenna applications.

Xiang-Yu CAO received the B.Sc and M.A.Sc degrees from the Air Force Missile Institute in 1986 and 1989, respectively. She joined the Air Force Missile Institute in 1989 as an assistant teacher. She became an associate professor in 1996. She received Ph.D. degree in the Missile Institute of Air Force Engineering University in 1999. From 1999 to 2002, she was engaged in postdoctoral research in Xi'an Electron Technology University, China. She was a Senior Research Associate in the Dept. of Electronic Engineering, City University of Hong Kong from June 2002 to Dec 2003. She is currently a professor of Telecommunication Engineering Institute, Air Force Engineering University. She has authored and coauthored more than 200 technical journal articles and conference papers, and holds ten China soft patent. She is the coauthor of two books entitled, Electromagnetic Field and Electromagnetic Wave, and Microwave Technology and Antenna published in 2007 and 2008, respectively. Her research interests include smart antennas, electromagnetic metamaterials and their antenna applications, and electromagnetic compatibility. 